\title{
Pólipos postinflamatorios y enfermedad inflamatoria intestinal. Reporte de caso y revisión de la literatura
}

\section{Literature Review and Case Report of Post-Inflammatory Polyps and Inflammatory Bowel Disease}

Hernando Marulanda, MD, ${ }^{1}$ William Otero, MD, ${ }^{2}$ Martín Gómez, MD. ${ }^{3}$

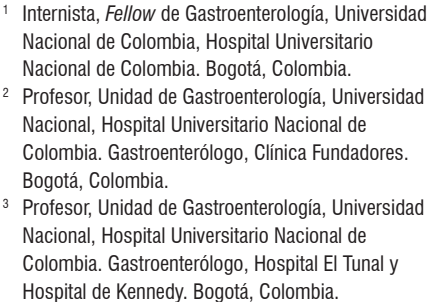

Internista, Fellow de Gastroenterología, Universidad Nacional de Colombia, Hospital Universitario Nacional de Colombia. Bogotá, Colombia.

2 Profesor, Unidad de Gastroenterología, Universidad Nacional, Hospital Universitario Nacional de Colombia. Gastroenterólogo, Clínica Fundadores. Bogotá, Colombia.

3 Profesor, Unidad de Gastroenterología, Universidad Nacional, Hospital Universitario Nacional de Colombia. Gastroenterólogo, Hospital El Tunal y Hospital de Kennedy. Bogotá, Colombia.

\begin{abstract}
Resumen
En pacientes con enfermedad inflamatoria intestinal (EII) de larga duración, pueden aparecer pólipos postinflamatorios o seudopólipos, los cuales se presentan en los sitios en donde previamente hubo inflamación severa. Se describe el caso de un paciente con colitis ulcerativa de 5 años de evolución, en quien se encontró una poliposis postinflamatoria generalizada durante la colonoscopia de control. Se hace una revisión del significado de esta alteración, su clasificación y de su tratamiento.
\end{abstract}

\section{Palabras clave}

Seudopolipos, pólipos postinflamatorios, colitis ulcerativa, inflamación.

\section{Abstract}

Post-inflammatory polyps or "pseudopolyps" may occur in patients who have long-term inflammatory bowel disease (IBD). They occur at sites where severe inflammation had previously occurred. We describe the case of a patient who had suffered from ulcerative colitis for five years when generalized post-inflammatory polyposis was discovered during a follow-upl colonoscopy. We review the meaning of this condition as well as its classification and treatment.

Key words

Pseudopolyps, post inflammatory polyps, ulcerative colitis, inflammation.

\section{INTRODUCCIÓN}

La colitis ulcerativa (CU) es una enfermedad autoinflamatoria que con la enfermedad de Crohn (EC) representan la inmensa mayoría de pacientes con enfermedad inflamatoria intestinal (EII) (1). Los objetivos actuales del tratamiento son la remisión de los síntomas clínicos y la cicatrización endoscópica de la mucosa (2). Con estos nuevos blancos terapéuticos (treat to target), cada día es más frecuente encontrar alteraciones endoscópicas estructurales residuales derivadas de la cicatrización y la reparación de la mucosa previamente afectada (3) y los seudopólipos son una de esas (4). Estos son neoformaciones de aspecto polipoide, conformados por tejido cicatricial en sitios de actividad inflamatoria previa, que reflejan la severidad de la misma a ese nivel (4). La denominación de seudopólipos hoy se considera obsoleta, ya que tanto histológica como macroscópicamente son verdaderos pólipos (5). La recomendación actual es que sean llamados pólipos postinflamatorios (6), que según su disposición pueden ser pólipos inflamatorios o pólipos filiformes (7). Son dos veces más frecuentes en CU que en EC (8). En general, no producen síntomas y casi siempre son hallazgos incidentales en los estudios radiológicos y endoscópicos que rutinariamente se realizan en el 
seguimiento de los pacientes con esta enfermedad (9). Sin embargo, pueden producir sangrado u obstrucción intestinal cuando alcanzan grandes tamaños (10). En estos últimos casos, el tratamiento es quirúrgico. Morfológicamente pueden simular una neoplasia.

En este artículo se presenta un paciente con colitis ulcerativa extensa, de 5 años de evolución, actualmente en remisión clínica y endoscópica, en quien se encontró poliposis postinflamatoria generalizada en un estudio de colonoscopia de control por diarreas recurrentes.

\section{CASO CLÍNICO}

Hombre de 42 años de edad, con diagnóstico de colitis ulcerativa extensa, de 5 años de evolución. Actualmente se encuentra en remisión clínica y endoscópica, con índice de Truelove y Witts (11) de 6 puntos. Cuando se hizo el diagnóstico inicial, pesaba $45 \mathrm{~kg}$ y su talla era $1,90 \mathrm{~m}$. Actualmente pesa $100 \mathrm{~kg}$. Está en tratamiento con mesalazina (MSLZ) en gránulos, $3 \mathrm{~g} /$ día asociada con azatioprina (AZA) en dosis de $1 \mathrm{mg} / \mathrm{kg} /$ día, desde hace 1 año. No recibe las dosis usuales recomendadas de AZA de 2,5 a 3 $\mathrm{mg} / \mathrm{kg}$ debido a que la MSLZ puede aumentar los niveles de AZA, con mayor riesgo de toxicidad (12). Por la severidad del cuadro clínico, al primer año del diagnóstico le propusieron terapia biológica con antifactor de necrosis tumoral (anti-FNT). Sin embargo, se optimizó el tratamiento de MSLZ adicionando enemas de la misma en dosis de 1 enema cada día y además prednisona en $40 \mathrm{mg}$ / día. Esta última progresivamente se fue disminuyendo después del primer mes, hasta suspenderla. Ha tenido varias recurrencias y en dos ocasiones se ha documentado infección por Clostridium difficile, tratada con metronidazol oral, y en la segunda ocasión con vancomicina oral en $125 \mathrm{mg}$, cuatro veces al día durante 10 días.

Actualmente se encuentra en remisión clínica, excepto por diarreas recurrentes secundarias a síndrome de intestino irritable. La calprotectina fecal es normal. El cuadro hemático y el perfil hepático son normales. En la colonoscopia total e ileoscopia de control solamente se observaron pólipos postinflamatorios. La evaluación de la mucosa colónica mostró un puntaje de Mayo de 0-1 (13) en los diferentes segmentos, y de manera generalizada pólipos postinflamatorios, más frecuentes en sigmoides y transverso, que se muestran en la figura 1 (A-D). Se tomaron múltiples biopsias de la mucosa colónica y de los pólipos postinflamatorios. El patólogo informó hiperplasia focal de criptas sin distorsión de su estructura ni infiltrados inflamatorios. Concluye que las alteraciones son cambios epiteliales reactivos residuales asociados con colitis crónica quiescente, lo cual se correlaciona con el proceso inflamatorio subyacente residual. Los hallazgos son característicos de fenómenos cicatriciales en la mucosa gastrointestinal. No se documentaron alteraciones que sugieran compromiso por lesiones premalignas o neoplásicas, lo que se correlaciona con la mayoría de los reportes en la literatura mundial.

El seguimiento endoscópico planteado fue el estándar de cuidado para la enfermedad inflamatoria intestinal.

\section{DISCUSIÓN}

El término pseudopoliposis fue acuñado por primera vez en 1974 por Appelman y colaboradores (14), para describir un síndrome clínico radiológico que afectaba con mayor frecuencia a pacientes con EII. El hallazgo imagenológico asociado afectaba principalmente al colon sigmoides, tratándose de numerosos defectos filiformes similares a gusanos, con un patrón de haustras normales (15).

Otros términos tales como pseudopoliposis gigante, poliposis inflamatoria gigante y poliposis filiforme han sido utilizados en la literatura para describir ese tipo de poliposis (16).

Los pólipos postinflamatorios son neoformaciones de aspecto polipoide presentes en los procesos cicatriciales de la mucosa, comprometida por inflamación severa de larga duración (17).

La patogénesis de estas estructuras no se conoce con exactitud (18). Sin embargo, se considera que pueden representar focos de proliferación celular en respuesta a una inflamación severa previa (19). La tracción mecánica y el peristaltismo progresivamente producen alargamiento y la coalescencia de varios focos de proliferación determinaría su tamaño final (20).

La mayor incidencia de estas alteraciones está presente en la CU con respecto a la EC. Se presume que se debe a una mayor inflamación de la mucosa en la CU (21). En esta última, la prevalencia va de un $12,5 \%$ a un $75 \%$ (22), aunque se encuentra más a menudo en las piezas de colectomía que en los exámenes endoscópicos $(21,22)$.

Su presencia aumenta con la extensión y la severidad de la enfermedad. Si bien su descripción más frecuente está limitada a la mucosa del colon, en la enfermedad de Crohn se han descrito en el esófago, en el estómago y en el intestino delgado (23).

Aunque la CU y la EC son las enfermedades inflamatorias más frecuentemente asociadas con los pólipos postinflamatorios, estos también han sido descritos en las colitis infecciosas, isquémicas y necrotizantes (24).

Con base en las características macroscópicas y la extensión de las lesiones, estos pólipos postinflamatorios se clasifican como se muestra en la tabla 1 (25).

A su vez, las lesiones gigantes han sido subclasificadas en las siguientes categorías (26):

- Seudopoliposis múltiple localizada

- Seudopoliposis gigante localizada 

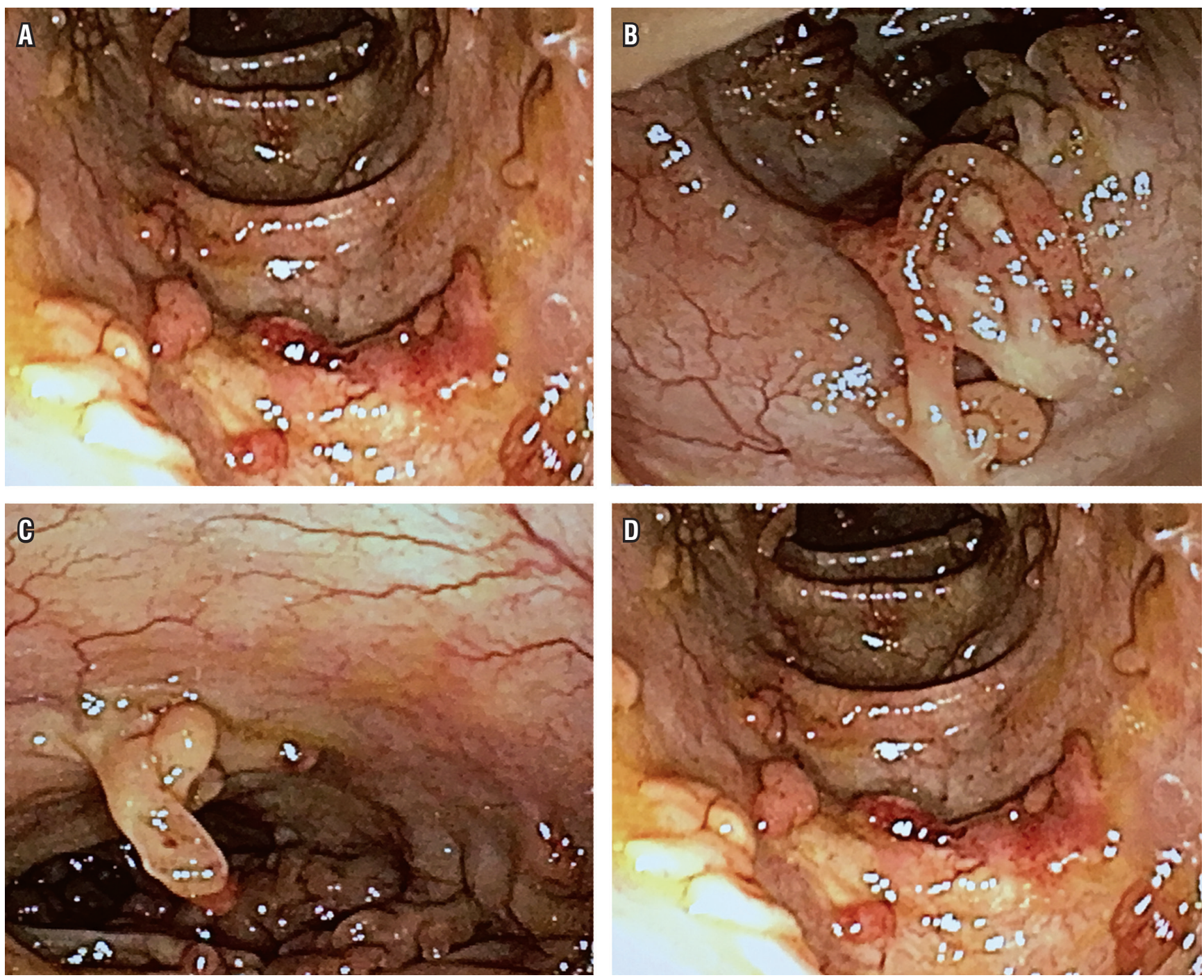

Figura 1. Pólipos postinflamatorios.

- Seudopoliposis generalizada

- Seudopoliposis digitiforme.

Tabla 1. Tipos de pólipos postinflamatorios

\begin{tabular}{ll}
\hline Poliposis generalizada & $\begin{array}{l}\text { Pólipos menores de } 5 \mathrm{~mm} \\
\text { generalizados }\end{array}$ \\
Poliposis múltiple localizada & $\begin{array}{l}\text { Pólipos menores de } 5 \mathrm{~mm} \text { focales } \\
\text { Pólipos delgados no lobulados de } \\
\text { distribución variable }\end{array}$ \\
Poliposis filiforme & $\begin{array}{l}\text { Pólipos de más de } 15 \mathrm{~mm} \text { de } \\
\text { distribución focal o difusa }\end{array}$ \\
\hline
\end{tabular}

La presentación clínica puede no variar de los síntomas propios de la EII, como son la presencia de diarrea, sangrado, anemia, pérdida de peso $y$, con gran frecuencia,

dolor abdominal (27). Sin embargo, en algunos casos se destaca la presencia de sensación de masa a nivel abdominal o la aparición de complicaciones dadas por la presencia y el tamaño de los pólipos, tales como la intususcepción o la obstrucción intestinal mecánica (28).

Se ha descrito que el tratamiento a largo plazo con MSLZ, esteroides y AZA puede disminuir el tamaño de estas lesiones, incluso desaparecerlas aunque sean gigantes (29).

Hay reportes recientes en los que se destaca el valor del uso de anti-FNT en el control de los focos de proliferación extracolónica, principalmente en el esófago, para aquellos pacientes con fracaso a la terapia inicial y síntomas relacionados persistentes (23-30).

Los pólipos están revestidos por mucosa colónica normal, edematosa o superficialmente ulcerada; el tallo a menudo es más fibrovascular, pero también puede contener fascí- 
culos de músculo liso. Los casos segmentarios o asociados con estenosis pueden confundirse con una neoplasia (31).

La gran mayoría de los preparados microscópicos muestra una marcada fibrosis, con la arquitectura histológica relativamente bien conservada (32). Es común encontrar hiperplasia de las criptas e inflamación focal inespecífica, infiltrado inflamatorio sobre la muscular de la mucosa, asociado con hiperplasia linfoide, hallazgos considerados generalmente de curso benigno (33). Los casos más severos se relacionan con erosiones, extensión transmural e hiperplasia linfática, que corresponden al intenso compromiso inflamatorio (34). Los segmentos no polipósicos son de gran importancia, ya que allí es donde frecuentemente se ha logrado documentar a los precursores de las lesiones neoplásicas (35).

$\mathrm{El}$ análisis inmunohistoquímico puede ser una herramienta complementaria, que utiliza anticuerpos anti-Ki67 $y$ anti-p53 en los fragmentos de tejido, con el fin de establecer un patrón anormal de proliferación celular y, por ende, el sustrato a patología tumoral (36).

La importancia de los pólipos postinflamatorios radica en que pueden correlacionarse con un mayor riesgo de cáncer de colon asociado con EII (CCAEII) o representar una lesión premaligna (37). Rutter y colaboradores (38) encontraron que el odds ratio (OR) de estas lesiones para CCAEII es de 2,29 (IC 95\%: 1,28-4,11) y Velayos y colaboradores (39) encontraron un riesgo similar, con un OR de 2,29 (IC 95\%: 1,28-4,11). Adicionalmente, en pacientes con CU de larga evolución, cuando hay pólipos postinflamatorios gigantes y displasia a su alrededor, el hallazgo puede confundirse con displasia asociada a masa $(38,39)$.

Además, en series de casos se ha establecido la relación existente entre el tamaño de los pólipos y la susceptibilidad de presentar alteraciones histológicas, encontrándose que los pólipos grandes demuestran ser adenomas frecuentemente $(40,41)$.

Por lo anterior, parece ser necesario plantear un seguimiento en un período más estrecho. Si bien no hay indicación de colectomía profiláctica para el manejo de estas lesiones, en el caso que los hallazgos endoscópicos, radiológicos o clínicos orienten hacia una neoplasia, la cirugía debe ser realizada para así obtener un estudio histopatológico completo que la descarte (42).

El manejo quirúrgico está indicada cuando las lesiones son gigantes y producen complicaciones mecánicas, así como también cuando hay displasia en las zonas adyacentes a las lesiones (43).

Actualmente no hay acuerdo sobre la forma como debe hacerse el seguimiento de estas lesiones. Por el momento debe mantenerse el estándar de cuidado de los pacientes con CU mediante colonoscopia y biopsias, pero, en este caso, de manera más minuciosa (44), con la recomendación de tomar biopsias tanto de los pólipos como de la mucosa adyacente a los mismos y adicionalmente con polipectomía aleatoria de algunas lesiones (45).

Algunos autores recomiendan intensificar el seguimiento endoscópico de los pacientes con pólipos postinflamatorios (46) y más aún en los pacientes con lesiones focales de gran tamaño (mayor de $15 \mathrm{~mm}$ ) teniendo como pauta al menos un control endoscópico anual (47).

\section{CONCLUSIÓN}

Los pólipos postinflamatorios son un hallazgo frecuente en pacientes con enfermedad inflamatoria intestinal (EII), en remisión clínica y endoscópica.

Se consideran consecuencia de la severa actividad inflamatoria subyacente. En la actualidad, su presencia no es considerada una lesión premaligna; sin embargo, hay reportes recientes que obligan a considerar un seguimiento endoscópico más estrecho, que incluya un estudio histopatológico de la lesión y de las zonas de mucosa sana adyacentes.

\section{REFERENCIAS}

1. Chen JH, Andrews JM, Kariyawasam V, et al. Review article: acute severe ulcerative colitis - evidence-based consensus statements. Aliment Pharmacol Ther. 2016;44 (2):127-44.

2. Peyrin-Biroulet L, Sandborn W, Sands BE, et al. Selecting therapeutic targets in inflammatory bowel disease (STRIDE): determining therapeutic goals for treat-to-target. Am J Gastroenterol. 2015;110:1324-38.

3. Christensen KR, Steenholdt C, Buhl SS, et al. Systematic information to health-care professionals about vaccination guidelines improves adherence in patients with inflammatory bowel disease in anti-TNFa therapy. Am J Gastroenterol. 2015;110(11):1526-32.

4. Sheikholeslami MR, Schaefer RF, Mukunyadzi P. Diffuse giant inflammatory polyposis: a challenging clinicopathologic diagnosis. Arch Pathol Lab Med. 2004; 128(11):1286-8.

5. Goldglaber MB. Pseudopolyposis in ulcerative colitis. Dis Colon Rectum. 1965:8;355-63.

6. Suzuki Y, Nakao K, Hishikawa E, et al. Filiform polyposis: inflammatory polyposis in ulcerative colitis, report of rare case. Stomach \& Intestine. 2001;36:585-9.

7. Renison DM, Forouhar FA, Levine JB, et al. Filiform polyposis of the colon presenting as massive hemorrhage: an uncommon complication of Crohn's disease. Am J Gastroenterol. 1983;78:413-6.

8. Balazs M. Giant inflammatory polyps associated with idiopathic inflammatory bowel disease. An ultrastructural study of five cases. Dis Colon Rectum. 1990;33:773-7.

9. Teague RH, Read AE. Polyposis in ulcerative colitis. Gut. 1975; 16:792-5.

10. Schneider R, Dickersin GR, Patterson JF. Localized giant pseudopolyposis. A complication of granulomatous colitis. Am J Dig Dis. 1973; 18:265-70. 
11. Goenka MK, Nag S, Kumar A, et al. Diagnosis of acute severe colitis. Trop Gastroenterol. 2014;35(Suppl 1):S1-8.

12. Gao X, Zhang FB, Ding L, et al. The potential influence of 5 -aminosalicylic acid on the induction of myelotoxicity during thiopurine therapy in inflammatory bowel disease patients. Eur J Gastroenterol Hepatol. 2012;24(8):958-64.

13. Schroeder KW, Tremaine WJ, Ilstrup DM. Coated oral 5-aminosalicylic acid therapy for mildly to moderately active ulcerative colitis: a randomized study. N Engl J Med. 1987;317:1625-9.

14. Appelman HD, Threatt BA, Ernst C, et al. Filiform polyposis of the colon: an unusual sequel of ulcerative colitis. Am J Clin Pathol. 1974;62:145-6.

15. Samach M, Train J. Demonstration of mucosal bridging in Crohn's colitis. Am J Gastroenterol. 1980;74:50-4.

16. Peh WC. Filiform polyposis in tuberculosis of the colon. Clin Radiol. 1988;39:534-6.

17. Dellinger GW, Lynch CA, Mikas AA. Colonic ganglioneuroma presenting as filiform polyposis. J Clin Gastroenterol. 1996;22:66-70.

18. Kelly JK, Gabos S. The pathogenesis of inflammatory polyps. Dis Colon Rectum. 1987;30:251-4.

19. Buck JL, Dachman AH, Sobin LH. Polypoid and pseudopolypoid manifestation of inflammatory bowel disease. Radiographics. 1991;11:293-304.

20. Loddo I, Romano C. Inflammatory bowel disease: genetics, epigenetics, and pathogenesis. Front Immunol. 2015;6:551.

21. Choi YS, Suh JP, Lee IT, et al. Regression of giant pseudopolyps in inflammatory bowel disease. 2012;6:240-3.

22. Dombal FT, Watts JM, Watkinson G, et al. Local complications of ulcerative colitis: stricture, pseudopolyposis, and carcinoma of colon and rectum. Br Med J. 1966;1:1442-7.

23. Soriani P, Tontini G, Neumann H, et al. Esophageal postinflammatory polyposis in extensive and severe Crohn's disease treated with anti-tumor necrosis factor alpha. Endoscopy. 2016;48(S 01):E261-2.

24. Hinrichs HR, Goldman H. Localized giant pseudopolyps of the colon. JAMA. 1968;205:248-9.

25. Tzkowitz SH, Yio X. Inflammation and cancer IV. Colorectal cancer in inflammatory bowel disease: the role of inflammation. Am J Physiol Gastrointest Liver Physiol. 2004;287:G7-17.

26. Freeman AH, Berridge FR, Dick AP, et al. Pseudopolyposis in Crohn's disease. Br J Radiol. 1978;51:782-7.

27. Mavrogenis G, Ngendahayo P, Kisoka P, et al. Intestinal obstruction caused by giant filiform polyposis in a patient with normal colon. Endoscopy. 2013;45(Suppl 2):UCTN: E80-1.

28. Lee CG, Lim YJ, Choi JS, et al. Filiform polyposis in the sigmoid colon: a case series. World J. Gastroenterol. 2010;16:2443-7.

29. Levine DS, Surawicz CM, Spencer GD, et al. Inflammatory polyposis two years after ischemic colon injury. Dig Dis Sci. 1986;31:1159-67.

30. Vainer B, Jess T, Andersen PS. Rapid tumour-like growth of giant filiform polyposis in a patient without a history of chronic bowel inflammation. APMIS. 2007;115(11):1306-10.
31. Oakley G, Schraut WH, Peel R, et al. Diffuse filiform polyposis with unique histology mimicking familial adenomatous polyposis in a patient without inflammatory bowel disease. Arch Pathol Lab Med. 2007;131:1821-4.

32. Raila FA, Athar M, Balaa MA. Colonic filiform polyposis and a malignant adenomatous polyp. South Med J. 1989;82(11):1448-9.

33. Sheikholeslami MR, Schaefer RF, Mukunyadzi P. Diffuse giant inflammatory polyposis: a challenging clinicopathologic diagnosis. Arch Pathol Lab Med. 2004;128:1286-8.

34. Macaigne G, Boivin JF, Cheaib S, et al. Polype filiforme unique développé dans un côlon normal et révélé par une hémorragie digestive sévère, Gastroenterol Clin Biol. 2006;30:913-5.

35. Yantiss RK, Oh KY, Chen YT, et al. Filiform serrated adenomas: a clinicopathologic and immunophenotypic study of 18 cases. Am J Surg Pathol. 2007;31:1238-45.

36. Rozenbajgier C, Ruck P, Jenss H, et al. Filiform polyposis: a case report describing clinical, morphological, and immunohistochemical findings. Clin Investig. 1992;70:520-8.

37. Rutter MD, Saunders BP, Wilkinson KH, et al. Thirty-year analysis of a colonoscopic surveillance program for neoplasia in ulcerative colitis. Gastroenterology. 2006;130:1030-8.

38. Rutter M, Saunders B, Wilkinson K, et al. Cancer surveillance in longstanding ulcerative colitis: endoscopic appearances help predict cancer risk. Gut. 2004;53:1813-6.

39. Velayos F, Loftus E, Jess T, et al. Predictive and protective factors associated with colorectal cancer in ulcerative colitis: a case-control study. Gastroenterology. 2006;130:1941-9.

40. Rutter M, Saunders B, Wilkinson K, et al. Severity of inflammation is a risk factor for colorectal neoplasia in ulcerative colitis. Gastroenterology. 2004;126:451-9.

41. Kusunoki M, Nishigami T, Yanagi H, et al. Occult cancer in localized giant pseudopolyposis. Am J Gastroenterol. 1992;87:379-81.

42. Okayama N, Itoh M, Yokoyama Y, et al. Total obliteration of colonic lumen by localized giant inflammatory polyposis in ulcerative colitis; report of a Japanese case. Int Med. 1996;35:24-9.

43. Katz S, Rosenberg RF, Katzca I. Giant pseudopolyps in Crohn's colitis. Am J Gastroenterol. 1981;76:267-71.

44. Esaki M, Matsumoto T, Fuyuno Y, et al. Giant inflammatory polyposis of the cecum with repeated intussusception in ulcerative colitis: report of a case. Am J Gastroenterol. 2009; 104:2873-4.

45. Maldonado TS, Firozzi B, Stone D, et al. Colocolonic intusscesption of a giant pseudopolyp in a patient with ulcerative colitis. Inflamm Bowel Dis. 2004;10(1):41-4.

46. Maggs JRL, Browning LC, Warren BF, et al. Obstructing giant post inflammatory polyposis in ulcerative colitis: Case report and review of the literature. J Crohns Colitis. 2008;2(2):170-80.

47. Boulagnon C, Jarezon JF, Diaz-Cives A, et al. Filiform polyposis: a benign entity? Case report and literature review. Pathol Res Pract. 2014;210:189-93. 